\title{
Blood-based miRNA as a biomarker in the diagnosis of bipolar II disorder
}

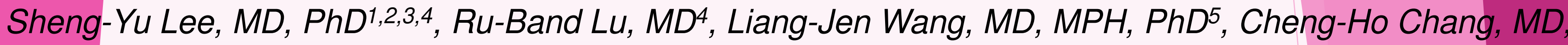 $M S^{1}$, Ti Lu, MD, MS'1, Tzu-Yun Wang, MD', Kuo-Wang Tsai, PhD6}

${ }^{1}$ Department of Psychiatry, ${ }^{6}$ Department of Medical Education and Research, Kaohsiung Veterans General Hospital, Kaohsiung, Taiwan; 2Department of Psychiatry, College of Medicine, National Yang-Ming University, Taipei, Taiwan; ${ }^{3}$ Department of Psychiatry, Faculty of Medi Kaohsiung Medical University Kaohsiung, Taiwan; ${ }^{4}$ Department of Psychiatry, College of Medicine and Hospital, National Cheng Kung Uni Tainan, Taiwan; ${ }^{5}$ Department of Child and Adolescent Psychiatry, Kaohsiung Chang Gung Memorial Hospital and Chang Gung University of Medicine, Kaohsiung, Taiwan;

Objectives: The diagnosis of Bipolar II disorder (BD-II) is currently based on patients' description of symptoms and clinical behavioral observations. This study explored the possibility of miRNA in peripheral blood (serum) as a specific blood-based biomarker for BD-II.

Methods: We developed six miRNA profiles using next-generation sequencing from samples taken from three randomly picked controls and patients with BD-II each from a total of 102 BD-II patients and 118 controls. We further selected six differentia expression miRNA candidates in the first cohort (as training group) of $79 \mathrm{BD}-\mathrm{II}$ and 95 controls and examined them using rea time PCR.

Results: We found that serum expression levels of miR-7-5p, miR-23b-3p, miR-142-3p, miR-221-5p, and miR-370-3p significantly increased in patients with BD-II compared with controls in the first cohort. The diagnostic power of identified miRNAs was analyzed using receiver-operating characteristic (ROC) curves; results revealed miR-7-5p (area under the curve [AUC], $0.728 ; p$ <0.0001), miR142-3p (AUC: 0.896; $p<0.0001$ ), miR-221-5p (AUC: 0.824; $p<0.0001$ ), and miR-370-3p (AUC: 0.703; $p<$ $0.0001)$ to be good biomarkers for diagnosis of BD-II. Support vector machine (SVM) measurements revealed that a combination of four miRNAs may further improve the diagnostic accuracy (AUC: 0.907). We further examined an independent testing group (BD-II, $n=20$; control, $n=20)$; the diagnostic power reached fair for BD-II (specificity = 90\% and sensitivity $85 \%)$.

Conclusion: We constructed miRNA panels using SVM, which may aid in the development of objective diagnostic tools for BD-II

Table 1. Clinicodemographic characteristics of participants

\begin{tabular}{|l|l|l|l|l|}
\hline & BD-ll & Healthy controls & $X^{2}$ or $\mathrm{t}$ & P value \\
\hline $\mathrm{n}$ & 102 & 118 & & \\
\hline $\begin{array}{l}\text { Age } \\
\text { SD) }\end{array}$ & & 3.3 & $<0.001$ \\
\hline Gender (M/ F) & $38 / 64$ & $61 / 57$ & 4.6 & 0.03 \\
\hline HAMD & $14.2 \pm 3.4$ & $\mathrm{~N} / \mathrm{A}$ & & \\
\hline YMIRS & $13.1 \pm 2.4$ & $\mathrm{~N} / \mathrm{A}$ & & \\
\hline
\end{tabular}

Figure 2. Small RNA profiles performed by Next-generation sequencing to identify candidate miRNAs
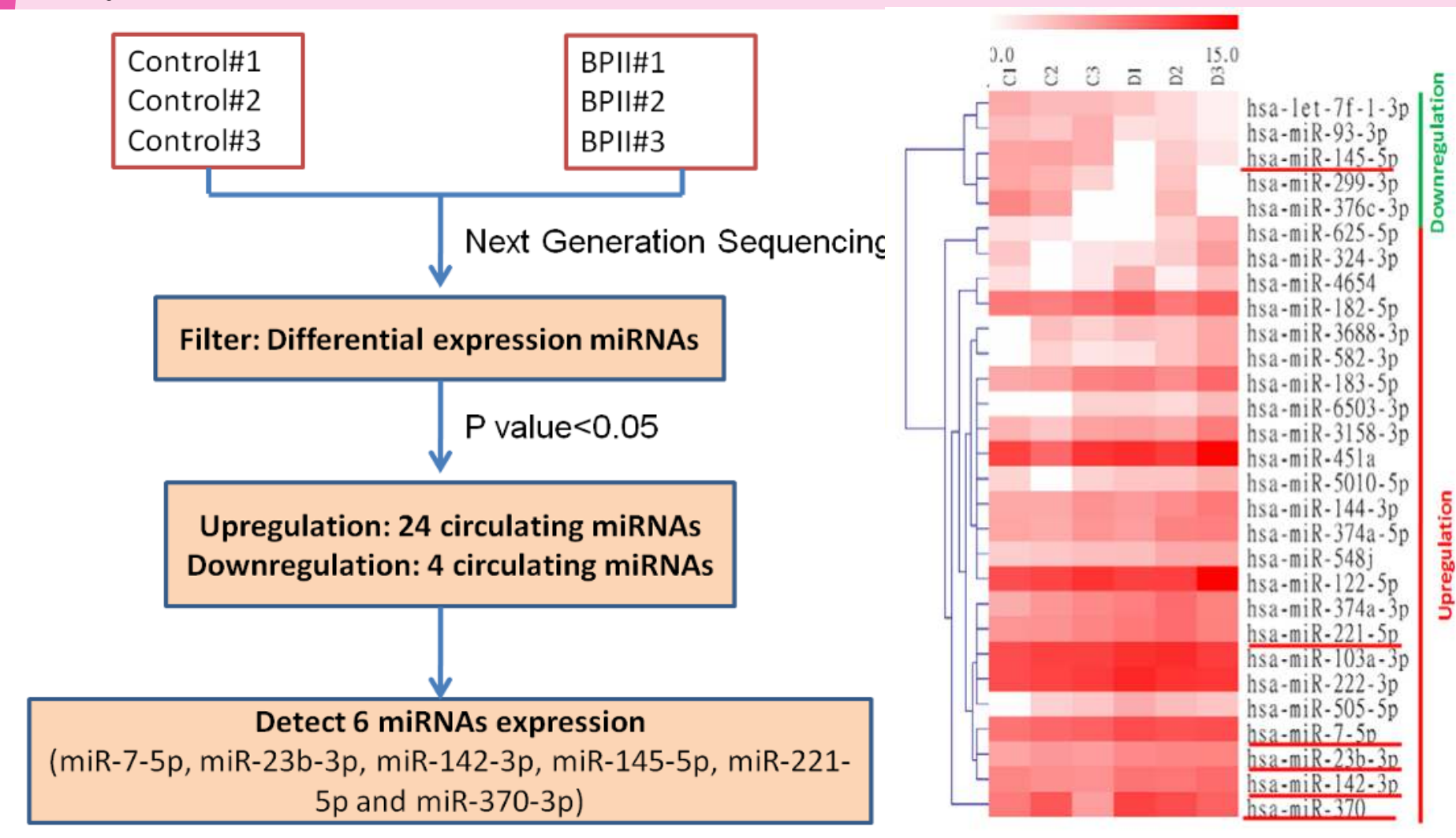

Figure 4. Diagnostic power of the six miRNA candidates using ROC curve analysis.

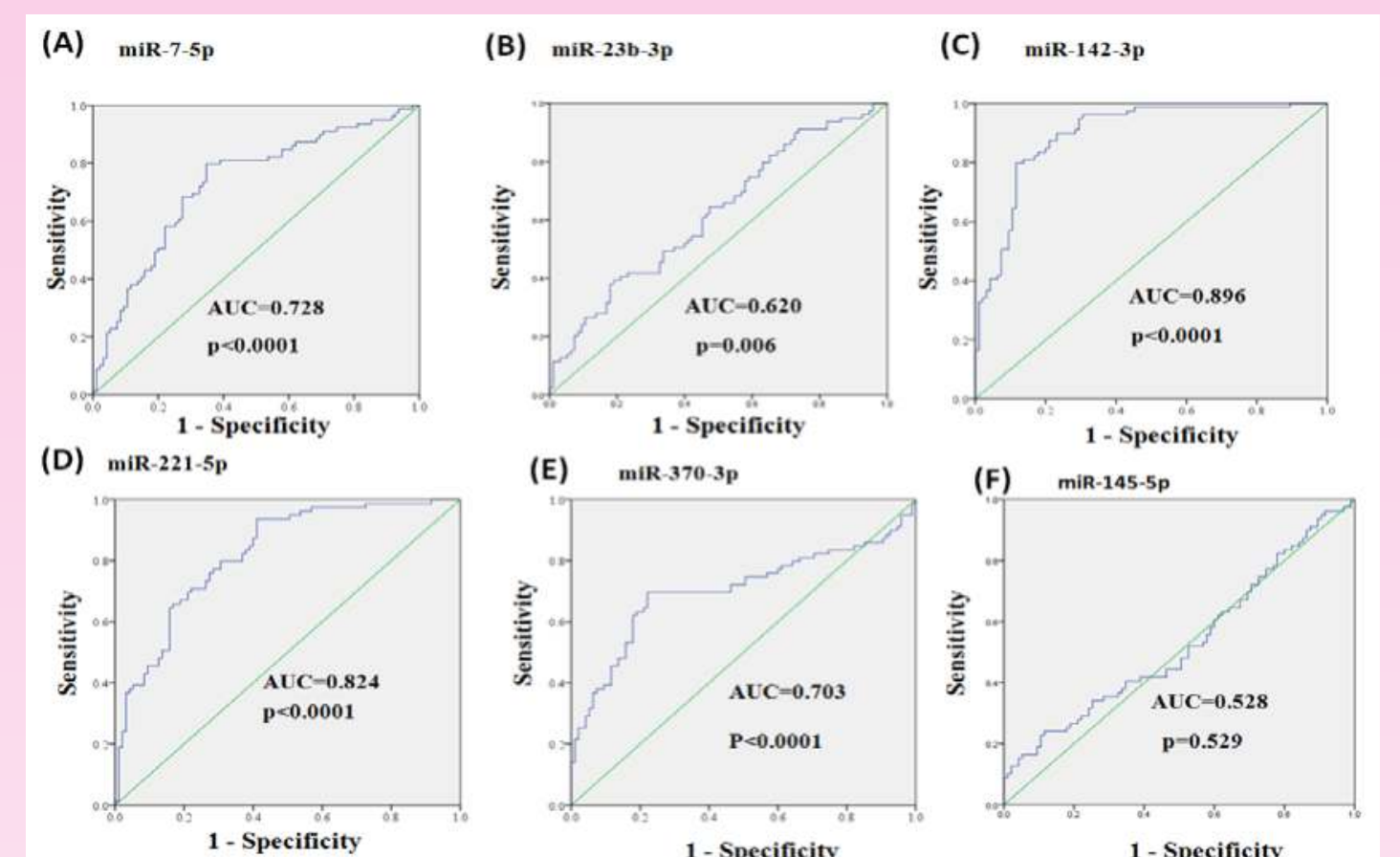

Figure 1. Flowchart to evaluate the potential of miRNA candidates for diagnostic biomarker.

\begin{tabular}{c|c} 
Identifying Cohort \\
BD-II $(\mathrm{N}=3)$ \\
Normal Controls $(\mathrm{N}=3)$
\end{tabular}$\quad \begin{gathered}\text { Training Cohort } \\
\mathrm{BD}-\mathrm{II}(\mathrm{N}=79) \\
\text { Normal Controls } \\
(\mathrm{N}=95)\end{gathered} \quad \begin{gathered}\text { Testing Cohort } \\
\mathrm{BD}-\mathrm{II}(\mathrm{N}=20) \\
\text { Normal Controls } \\
(\mathrm{N}=20)\end{gathered}$

Figure 3. Expression levels of circulating miRNAs in serum in BD-II and healthy controls using real-time PCR
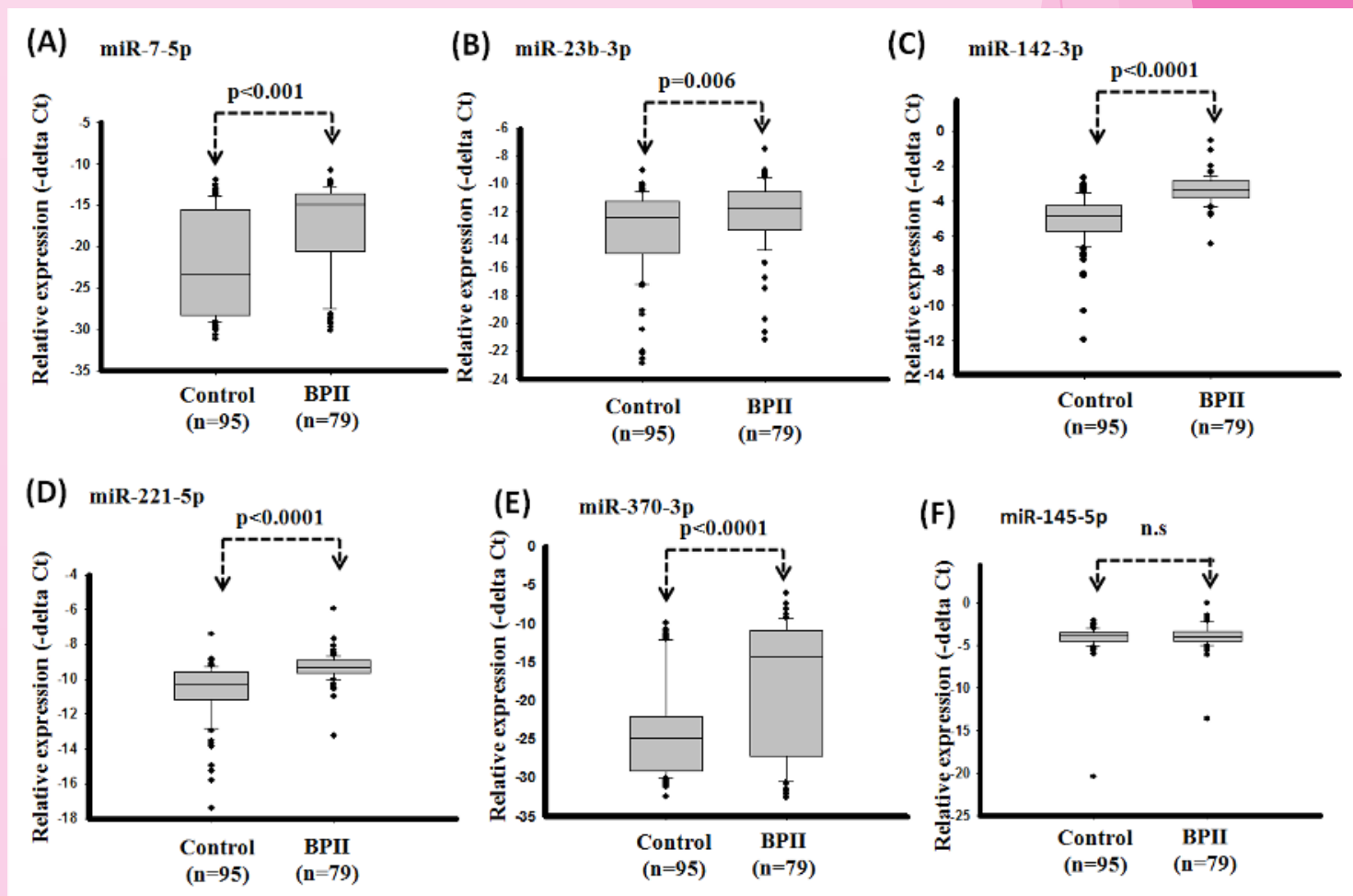

Figure 5. Building of a diagnostic panel for BD-II using SVM model.

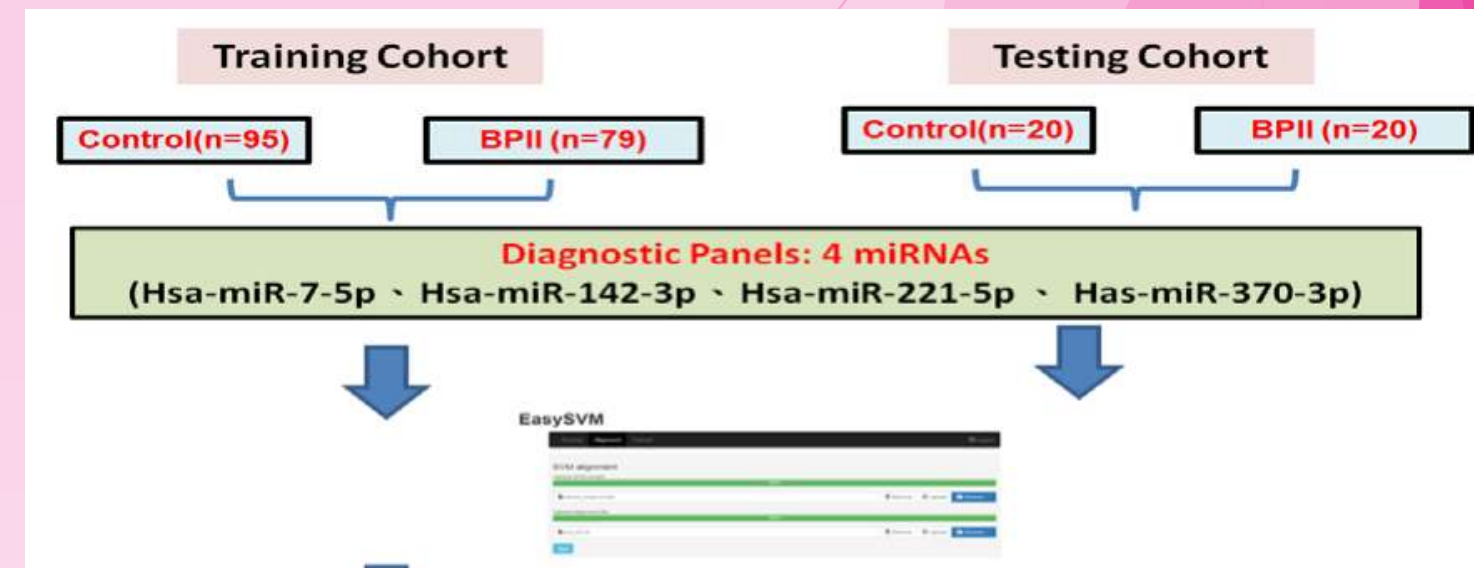

